\title{
Vorticity Transports in Wall Turbulent Flow Under Spanwise Wall Jet Forcing and Blowing-Suction Control
}

\author{
Yong JI and Xi CHEN ${ }^{\mathrm{b}, 1}$ \\ ${ }^{a}$ College of Science, Harbin Institute of Technology (Shenzhen), Shenzhen 518055, \\ China \\ ${ }^{\mathrm{b}}$ Key Laboratory of Fluid Mechanics of Ministry of Education, Beihang University, \\ Beijing, China
}

\begin{abstract}
This study investigates vorticity transports in wall turbulent flow under blowing-suction (BS) and spanwise opposed wall jet forcing (SOJF) control via direct numerical simulation of the Navier-Stokes equation. For combining SOJF and BS control, the drag reduction can achieve maximum value about $33 \%$ - obviously larger than individual control (18\% for SOJF and $27 \%$ for BS). Following Ji et al. [1], the mean spanwise vorticity $\left(\Omega_{Z}\right)$, the vorticity fluctuation transports in spanwise direction $\left(-v^{\prime} \omega_{z}{ }^{\prime}\right)$ and normal direction $\left(w^{\prime} \omega_{y}{ }^{\prime}\right)$ are investigated. Results support our previous conclusion that the frictional drag is considerably contributed by the transports of vorticity fluctuations. A triple decomposition (mean, coherent and random) shows that the role of the random $-v^{\prime \prime} \omega_{z}{ }^{\prime \prime}$ is drag adding, but other terms - random $w^{\prime \prime} \omega_{y}{ }^{\prime \prime}$ and the coherent $-\tilde{v} \widetilde{\omega}_{z}$ and $\widetilde{w} \widetilde{\omega}_{y}$ transports - are all drag decreasing.
\end{abstract}

Keywords. Turbulent boundary layer, Drag reduction, SOJF, Blowing and suction.

\section{Introduction}

Wall turbulence induces much skin friction drag that contributes approximately $90 \%$ of the total drag of underwater vehicles and $50 \%$ of commercial aircraft. It is no doubt that the turbulent flow near the wall must be controlled for drag reduction. According to whether extra energy is required, the control strategies are grouped into passive and active categories[2]. For passive control, riblets and superhydrophobic surfaces are two kinds of common controls which can yield significant drag reductions[3-6]. On the other hand, blowing and suction [7], as well as streamwise traveling wave-based strategies[811] are active controls resulting in considerable drag reductions. However, the application of these methods is obstructed by various problems including high powerinput, sophisticated sensors and actuators, or difficulties in wall-surface sustenance[12, 13].

The study of drag reduction mechanisms keeps research hot in recent years that notable progress has been made. One remarkable finding of recent study is that the skin friction is not only related to the inner flow of the turbulent boundary layer, but also outer

\footnotetext{
${ }^{1}$ Corresponding Author, Xi CHEN, Key Laboratory of Fluid Mechanics of Ministry of Education, Beihang University, Beijing, China; Email: chenxi97@outlook.com.
} 
large-scale vortices which generates skin friction growing with the Reynolds number [14]. This finding implies that, in the turbulent boundary layer, drag reduction can be realized by manipulating large-scale coherent structures which are far away from wall. Schoppa and Hussain[15] suggested imposing large-scale counter-rotating streamwise swirls to reduce drag, and $20 \%$ drag reduction was obtained in a turbulent channel at $\operatorname{Re}_{\tau} \approx 100$. Furthermore, Yao et al. $[10,11]$ developed the large-scale drag control concept works by using the near-wall spanwise opposed wall jet forcing (SOJF) and obtained inspiring drag reduction for $\mathrm{Re}_{\tau}$ up to 550 .

Several frame works have been put forward to analyse decompose scheme of skin friction drag based on different flow motions. For example, Fukagata et al.[16] first derived well known FIK identity that builds relation between the Reynolds shear stress and the skin-friction coefficient; Renard \& Deck[17] proposed an alternative expression for $C_{f}$ by considering the effect of production on drag; Chen et al. [18] start from energy budget analysis and identified roles of dissipative structures on skin friction. Recently, Ji et al.[1] derived the formula that links the drag coefficient and the motion of the vortical structures, and applied new formula to analyse channel flow under SOJF control.

The present work makes direct numerical simulation (DNS) about channel flow with SOJF and BS control which yields $33 \%$ drag reduction. Then, the DNS data is analysed by using vortical formula developed in [1]. The results is consistent with previous conclusion that the random spanwise-vorticity transport $\left(-v^{\prime \prime} \omega_{z}^{\prime \prime}\right)$ is only term contributing to drag which should be suppressed for more effective drag reduction.

\section{Computational Approach and Drag Decomposition}

\subsection{Control Schemes}

The simulation is based on the incompressible Navier-Stokes equations as follow,

$$
\begin{aligned}
& \partial u_{i} / \partial x_{i}=0 \\
& \partial_{t} u_{i}+\partial_{j} u_{i} u_{j}=v \partial_{j} \partial_{j} u_{i}-\partial_{i} p / \rho+F_{i} .
\end{aligned}
$$

For SOJF, a spanwise forcing is added [11]:

$$
F_{z}=A_{s} \sin (\beta z) g(y)
$$

where $A_{s}$ is the forcing amplitude, $\beta(=2 \pi / \lambda)$ is the spanwise wavenumber, and $g(y)$ is a dimensionless forcing as a function of $y$. In this paper, all control parameters are nondimensionalized by viscous units of uncontrolled flow(indicated by the subscript 0 ): a) $A_{s}^{+}=A_{s} v / u_{\tau, 0}^{3}$; b) $\lambda^{+}=2 \pi / \beta^{+}\left(\beta^{+}=\beta v / u_{\tau, 0}\right)$; and c) $y^{+}=y u_{\tau, 0} / v$. The forcing function $g\left(y^{+}\right)$in Eq. (3) is $g\left(y^{+}\right)=y^{+} \exp \left(-\eta y^{+2}\right)$, where the decay factor $\eta$ specifies the wall-jet velocity profile in the wall-normal direction. The function $g$ has its maximum at $y^{+}=y_{c}^{+}=1 / \sqrt{2 \eta}$, corresponding to the height of the spanwise wall-jet maximum velocity. The function $g$ is normalized by its maximum value. In addition, 
blowing and suction is applied at bottom and top wall of channel. According to Han\&Huang[19], we set $\left.v\right|_{\text {wall }}=-\left.v\right|_{y^{+}=15}$, where $v$ normal velocity at wall.

\subsection{Simulation Parameters}

The open-source finite difference software "Incompact3d" [20] is used to realize control strategies. The no-slip conditions are applied at the walls, and the periodicity in the streamwise and spanwise directions is imposed. In wall normal direction, sixth-order compact schemes are used based on a stretched Cartesian grid. A low-storage third order Runge-Kutta scheme is used to perform time integration. A constant flow rate is set and the bulk velocity $U_{b}$ is maintained at a constant value by a procedure that adjusts the mean pressure gradient. The simulated Reynolds number $\mathrm{Re}_{\tau}$ is 180 . The $\mathrm{SOJF}$ parameters are set as same with Ji et al.[1]. The $C_{f}$ at the bottom wall is used to calculate drag reduction $\mathrm{R}$,

$$
R=\left(1-C_{f}^{\text {control }} / C_{f}\right) \times 100 \%
$$

More information about simulation is listed in table 1.

Table 1. Details of the present direct numerical simulations

\begin{tabular}{ccccccc}
\hline $\mathrm{Re}_{\tau}$ & $\mathrm{Re}_{b}$ & $\mathrm{~T}$ & $L_{x} / h, L_{z} / h$ & $L_{x}{ }^{+}, L_{z}^{+}$ & $\mathrm{N}_{\mathrm{x}} \times \mathrm{N}_{\mathrm{y}} \times \mathrm{N}_{z}$ & $\mathrm{R}$ \\
\hline 180 & 2800 & 800 & $4 \pi, 2 \pi$ & 2264,1134 & $192 \times 129 \times 128$ & $27 \%(\mathrm{BS}), 33 \%(\mathrm{BS}+\mathrm{SOJF})$ \\
\hline
\end{tabular}

\subsection{Drag Decomposition}

According to Ji et al. [1], the drag coefficient can be decomposed as follow,

$$
C_{f}=\underbrace{\int_{0}^{2} \frac{\varepsilon}{\varepsilon_{*}} d y^{\prime}}_{C_{\Omega}}+\underbrace{\int_{0}^{2} \overline{\frac{-v^{\prime} \omega_{z}^{\prime}}{u}}}_{T_{Z}} d y^{\prime}+\underbrace{\int_{0}^{2} \frac{\overline{-w^{\prime} \omega_{y}^{\prime}} \bar{u}}{\varepsilon_{*}} d y^{\prime}}_{T_{Y}}
$$

Furthermore, introducing the triple decomposition,

$$
u_{i}^{\prime}=\bar{u}_{i}+\tilde{u}_{i}+u_{i}^{\prime \prime}, \quad u_{i}^{\prime \prime}=u_{i}^{\prime}-\tilde{u}_{i}
$$

where $\tilde{u}_{i}$ is the coherent part, and $u_{i}^{\prime \prime}$ is the random turbulence part, satisfying $\overline{\tilde{u}}_{i}=0$, $\overline{u_{i}^{\prime \prime}}$ and $\tilde{u}_{i}^{\prime \prime}=0$. The triple-decomposed Eq. (5) formula is:

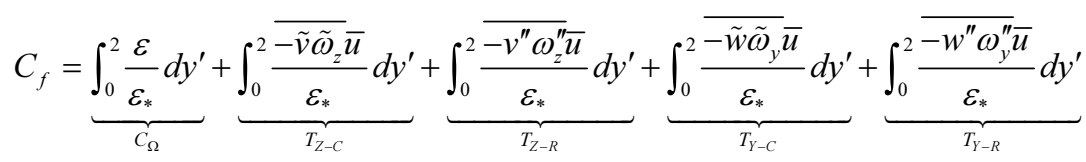


where the sub-scripts $C, R$ indicate coherent and random motions, respectively. Using Eq. (7), we can separate the drag contributions by other vortical motions and by the largescale swirls resulted from control.

\section{Results}
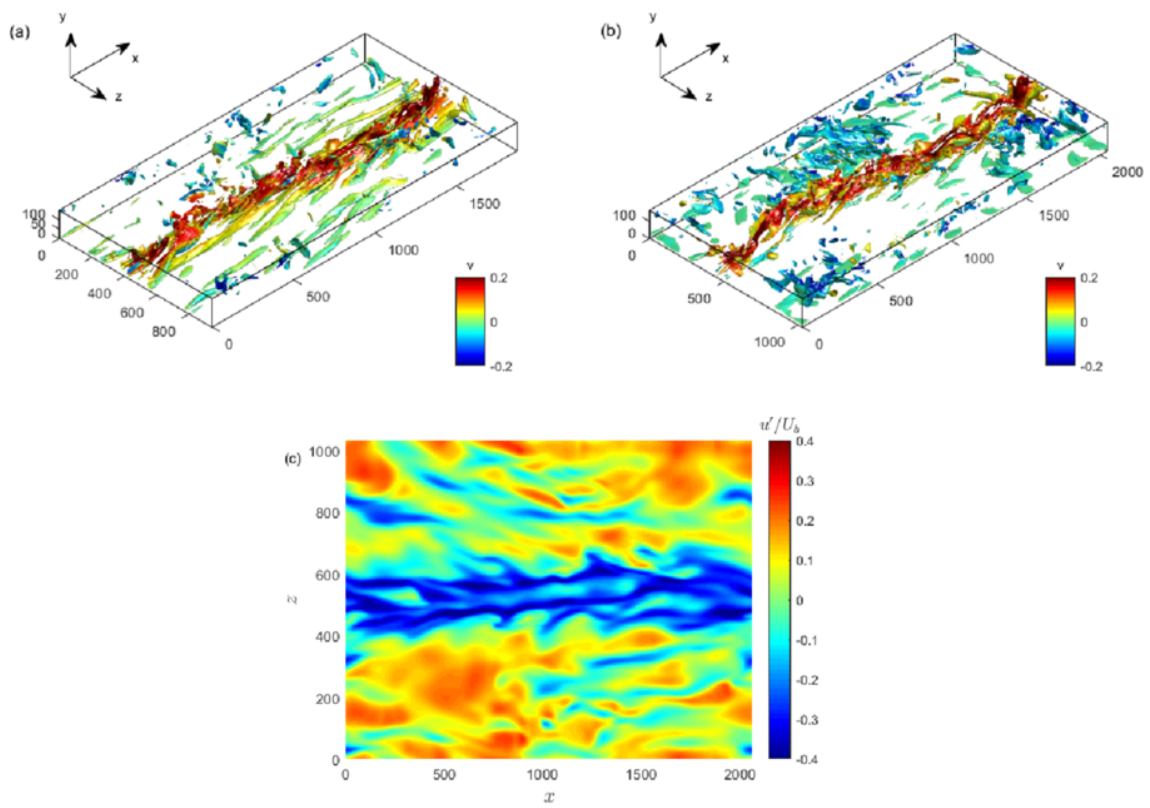

Figure 1. The iso-surface of instantaneous weighted wall-normal $\left(\bar{u} w^{\prime} \omega_{y}{ }^{\prime}\right)$ (a) and spanwise $\left(-\bar{u} v^{\prime} \omega_{z}{ }^{\prime}\right)(\mathrm{b})$ vorticity transport or dispersion in Eq. (5); the color bar denotes $v / U_{b}$ ranging from -0.2 to 0.2 . Contour of streamwise velocity fluctuations in the $(x-z)$ plane at $y^{+}=20$ (c). Note that for (a) the iso-surface value is 0.1 and for (b) corresponding value is 0.7 , the color bar for (c) ranges from -0.4 to 0.4 .

Figure 1(a) shows the three-dimensional(3D) iso-surface of weighted wall-normal vorticity transport or dispersion given by equation (5) in channel under the BS+SOJF control. It is obvious that, under the large scale spanwise control, the fluctuated structures mostly concentrate near the middle region of channel, which is different from no-control case. These structures contribute much on wall-normal vorticity transport or dispersion among the whole channel, because they induce intense velocity fluctuation increasing the transport rate. Figures 1(b) further displays the 3D iso-surface of weighted spanwise vorticity transport or dispersion $-\bar{u} v^{\prime} \omega_{z}{ }^{\prime}$ which is similar with the distributions of $\bar{u} w^{\prime} \omega_{y}{ }^{\prime}$. This is reasonable because the $\omega_{z}$ can also be generated by spanwise vortices tilted from the quasi-streamwise vortices generated near the middle-span. Figure 1(c) shows that there is low speed region in cross plane central, which is formed by merger of streamwise streaks under the action of the opposed wall-jet.

The time and $x$-axis averaged patterns of $\bar{u} w^{\prime} \omega_{y}{ }^{\prime}$ and $-\bar{u} v^{\prime} \omega_{z}{ }^{\prime}$ in cross-section view are shown in figure 2 (a) and figure $2(\mathrm{~b})$. The cross-plane velocity vectors $(\widetilde{w}, \tilde{v})$ are also displayed. It is obvious that SOJF results in upward flow motions distributing at both sides of the middle of the channel. As a result, the streamwise velocity at the central 
is slower than at the flank with the same height, which can be seen from figure 2(c). The value of $\left\langle\bar{u} w^{\prime} \omega_{y}{ }^{\prime}\right\rangle_{x t}$ is negative across all $y-z$ plane, but the value of $\left\langle-\bar{u} v^{\prime} \omega_{z}{ }^{\prime}\right\rangle_{x t}$ is negative at most region but positive in the jet collapsing region $\left(0.5<z / L_{z}<1.5\right.$ and $0<$ $y / H<0.4)$. Compared to results shown in reference [1], the intense $\left\langle\bar{u} w^{\prime} \omega_{y}{ }^{\prime}\right\rangle_{x t}$ and $\left\langle-\bar{u} v^{\prime} \omega_{z}{ }^{\prime}\right\rangle_{x t}$ are little weaker but still occurs at the flank of the streak envelop where $d \bar{u} / d y$ changes dramatically along the spanwise direction.
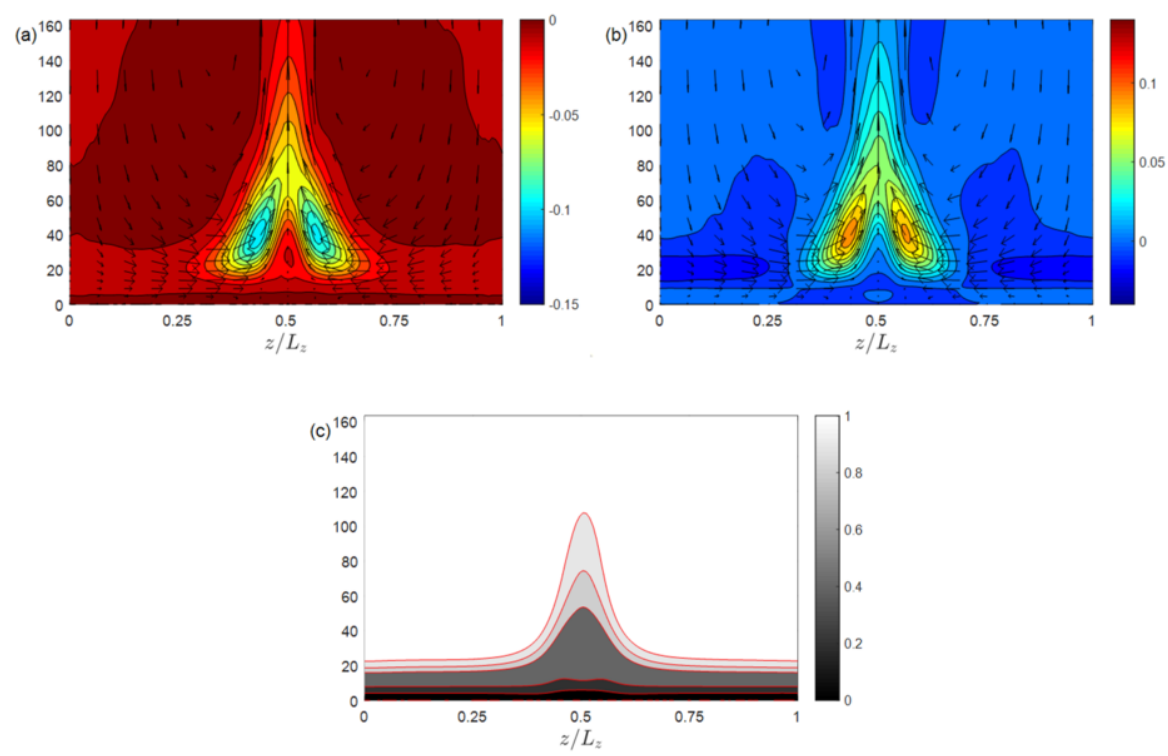

Figure 2. Cross-plane $(y-z)$ view of the mean velocity field $\left(\tilde{u}\right.$, normalized by $\left.U_{b}\right)$ with the contours of $\bar{u} w^{\prime} \omega_{y}{ }^{\prime}(\mathrm{a})$ and $-\bar{u} v^{\prime} \omega_{z}{ }^{\prime}(\mathrm{b})$ in wall unit, and (c) is the contours of streamwise $\langle u\rangle_{x t}$ showing the envelop of streaks. Color bar for (a) ranges from 0 to -0.15 , for (b) ranges from 0.14 to -0.04 , for (c) ranges from 0 to 1 .

Figure 3 shows the wall normal profiles of different $C_{f}$ components under the BS and BS+SOJF control. Figure 3(a) shows that the BS control leading to a bulge structure at about $y^{+}=10$, especially for $C_{\Omega}$ (blank dashed line) and $T_{Z}$ (blue dashed line). After adding SOJF control, this bulge structure impaired by spanwise jet (see black and blue solid line in figure 3(a)). Furthermore, the trip decomposition components under $\mathrm{BS}+\mathrm{SOJF}$ control are shown in figure 3(b) and 3(c). The profiles of $T_{Y}$ and $T_{Y-C, R}$ are similar with previous result[1] that $T_{Y}$ is always negative and coherent part $T_{Y-C \text {, }}$ contributes most part of $T_{Y}$ with a maximum location $y^{+} \sim 30$. On the contrary, the behaviour of $T_{Z}$ near wall is obviously different from result in [1] that the $T_{Z-R}$ first increases and then decreases and the maximum location of $T_{Z}$ is more outer than the case with only SOJF control.

Figure 4(a) shows the quantitative contribution to $C_{f}$ that the red color denotes $C_{\Omega}$ contribution and the blue color denotes $T_{Y}+T_{Z}$ contribution. Before control, $C_{\Omega}$ is $61 \%$ and $T_{Y}+T_{Z}$ is $39 \%$; after BS control $C_{\Omega}$ is $50 \%$ and $T_{Y}+T_{Z}$ is $23 \%$; after $\mathrm{BS}+\mathrm{SOJF}$ control, $C_{\Omega}$ is $53 \%$ and $T_{Y}+T_{Z}$ is $14 \%$. The BS control decreases $C_{\Omega}$ and $T_{Y}+T_{Z}$ simultaneously, but BS+SOJF control mainly decreases $T_{Y}+T_{Z}$ and increases $C_{\Omega}$ compared to BS control. Figure 4(b) show the coherent and random decomposition of $T_{Y}$ (left bar) and $T_{Z}$ (right bar) for BS and BS+SOJF control, respectively. It is reasonable 
that BS control only has random components. The random component $T_{Z-R}$ is only term contributing to drag under $\mathrm{BS}+\mathrm{SOJF}$, which is consistent with result of [1].
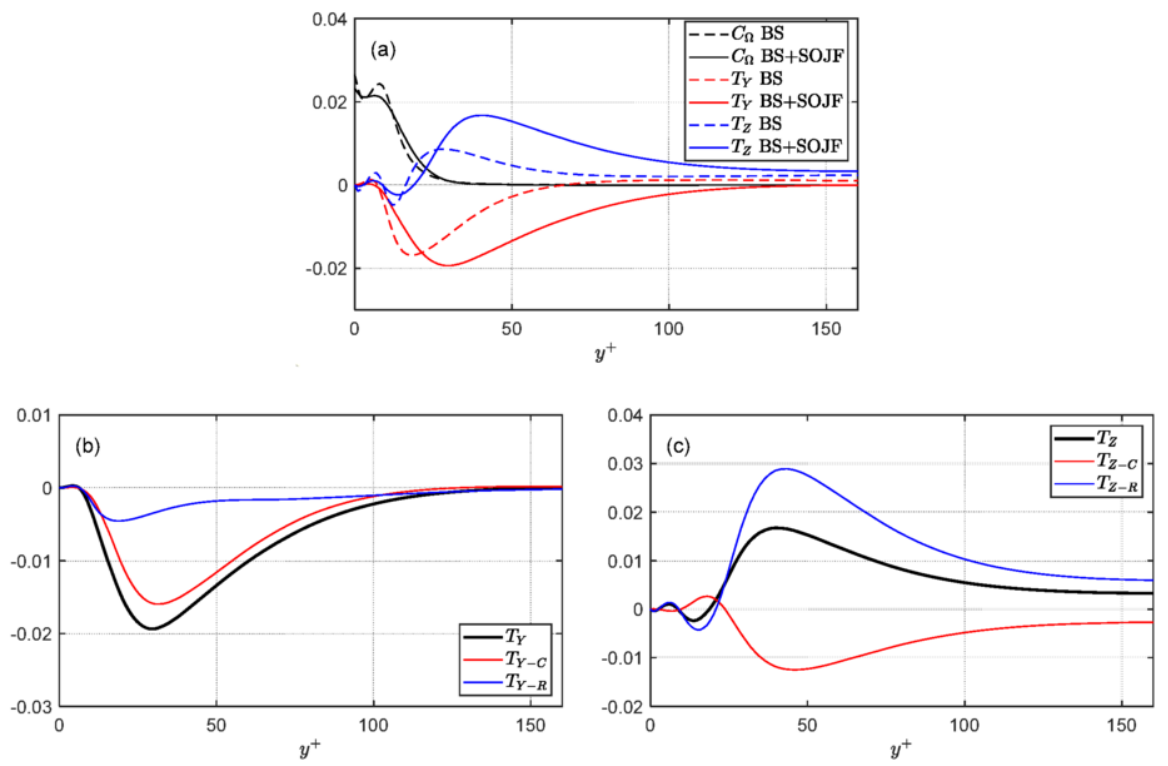

Figure 3. (a) Wall-normal profiles of $C_{\Omega}, T_{Y}$ and $T_{Z}$ defined in Eq. (5); Solid lines - BS+SOJF control; dashed lines - only BS control. (b) Wall-normal profiles of $T_{Y}, T_{Y-R}$ and $T_{Y-C}$ defined in Eq. (7). (c) wall-normal profiles of $T_{Z}, T_{Z-R}$ and $T_{Z-C}$ defined in Eq. (7)
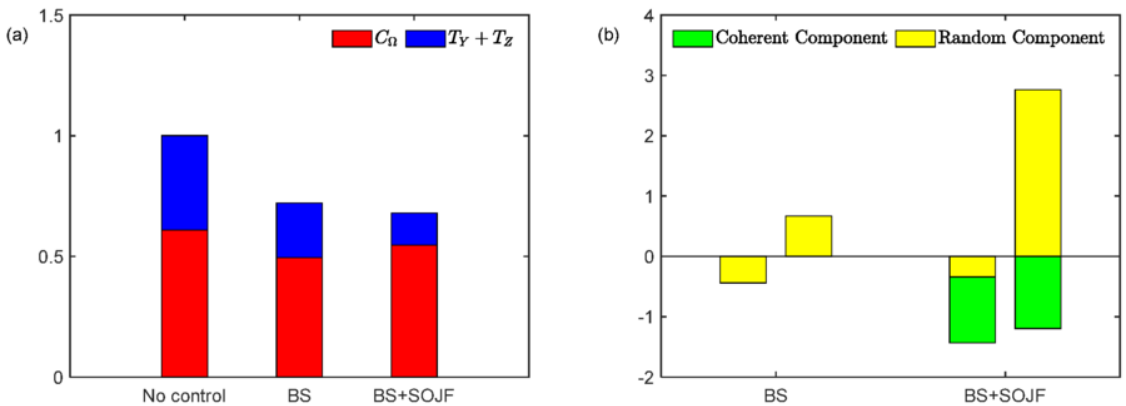

Figure 4. (a) Bar chats of $C_{\Omega}, T_{Z}$ and $T_{Y}$ for uncontrolled and controlled cases. (b) Triple decomposed $T_{Y-R}$ and $T_{Y-C}$ (left bar), $T_{Z-R}$ and $T_{Z-C}$ (right bar).

\section{Conclusion}

Following the idea of composite active drag control [21], we investigate the vorticity transport in turbulent channels under BS and BS+SOJF active control. The decomposition of friction coefficient based on vorticity transport is examined. We find that $\mathrm{BS}+\mathrm{SOJF}$ control perform a better drag reduction (33\%) compared to individual BC control $(27 \%)$ or individual SOJF control $(18 \%)$. Under the BS+SOJF control, the contribution of different vorticity transport terms to total drag coefficient is similar with 
our previous result [1] that $T_{Z-R}$ is only term in charge of drag friction, other terms play a role in drag reduction. This analysis suggests that the suppression of random spanwisevorticity transport is still the target for effective drag reduction under composite control including SOJF.

\section{References}

[1] Ji, Yong \& Yao, Jie \& Hussain, Fazle \& Chen, Xi. Vorticity transports in turbulent channels under largescale control via spanwise wall jet forcing. Physics of Fluids. 33. 095112(2021).

[2] M. Gad-el Hak, Flow control: passive, active, and reactive flow management(Cambridge University Press, 2007).

[3] J. P. Rothstein, Slip on superhydrophobic surfaces, Annual Review of Fluid Mechanics, 89285(2010).

[4] H. Park, G. Sun, et al., Superhydrophobic turbulent drag reduction as a function of surfacegrating parameters, Journal of Fluid Mechanics 747, 722 (2014).

[5] A. Checco, B. M. Ocko, A. Rahman, C. T. Black, M. Tasinkevych, A. Giacomello, and S. Di-etrich, Collapse and reversibility of the superhydrophobic state on nanotextured surfaces, Physical Review Letters 112, 216101 (2014).

[6] A. Rastegari and R. Akhavan, The common mechanism of turbulent skin-friction drag reduc-tion with superhydrophobic longitudinal microgrooves and riblets, Journal of Fluid Mechanics 838, 68 (2018).

[7] H. Choi, P. Moin, and J. Kim, Active turbulence control for drag reduction in wall-bounded flows, Journal of Fluid Mechanics 262, 75 (1994).

[8] W. Jung, N. Mangiavacchi, and R. Akhavan, Suppression of turbulence in wall-bounded flowsby highfrequency spanwise oscillations, Physics of Fluids A: Fluid Dynamics 4, 1605 (1992).

[9] A. Yakeno, Y. Hasegawa, and N. Kasagi, Modification of quasi-streamwise vortical structure in a dragreduced turbulent channel flow with spanwise wall oscillation, Physics of Fluids 26, 085109 (2014).

[10] J. Yao, X. Chen, F. Thomas, and F. Hussain, Large-scale control strategy for drag reduction in turbulent channel flows, Physical Review Fluids 2 (2017).

[11] J. Yao, X. Chen, and F. Hussain, Drag control in wall-bounded turbulent flows via spanwise opposed wall-jet forcing, Journal of Fluid Mechanics 852, 678 (2018).

[12] M. E. Lozier, F. O. Thomas, and S. Gordeyev, AIAA Scitech 2020 Forum.

[13] F. O. Thomas, T. C. Corke, M. Iqbal, A. Kozlov, and D. Schatzman, Optimization of dielectricbarrier discharge plasma actuators for active aerodynamic flow control, Aiaa Journal 47, 2169(2009).

[14] M. Quadrio, Drag reduction in turbulent boundary layers by in-plane wall motion, Philosophical Transactions of the Royal Society A, 1428 (2011).

[15] W. Schoppa and F. Hussain, A large-scale control strategy for drag reduction in turbulent boundary layers, Physics of Fluids 10, 1049 (1998).

[16] K. Fukagata, K. Iwamoto, and N. Kasagi, Contribution of reynolds stress distribution to the skin friction in wall-bounded flows, Physics of Fluids 14, 73 (2002).

[17] N. Renard and S. Deck, A theoretical decomposition of mean skin friction generation into physical phenomena across the boundary layer, Journal of Fluid Mechanics 790, 339 (2016).

[18] X. Chen, J. Yao, and F. Hussain, Theoretical framework for energy flux analysis of channels under drag control, Phys. Rev. Fluids 6, 013902 (2021).

[19] B.-Z. Han and W.-X. Huang, Active control for drag reduction of turbulent channel flow based on convolutional neural networks, Phys. Fluids 32, 095108 (2020).

[20] S. Laizet and E. Lamballais, High-order compact schemes for incompressible flows: A simple and efficient method with quasi-spectral accuracy, Journal of Computational Physics 228, 5989 (2009).

[21] J. Yao, X. Chen, and F. Hussain, Composite active drag control in turbulent channel flows, Phys. Rev. Fluids 6, 054605(2021). 